\title{
NONLINEAR DETERMINISTIC MODELLING OF ECOLOGICAL SCENARIOS - TYPICAL PREDICTIONS AND LIMITATIONS
}

\author{
Torsten Lindström \\ Michael Lindberg \\ Diauddin Nammari \\ Willem Stolte \\ University of Kalmar, Sweden
}

\begin{abstract}
Modelling population and community dynamics has evolved a lot after the early attempts by Malthus (1798), Darwin (1878), Lotka (1925), and Volterra (1926). The power of nonlinear simple deterministic models is usually that they show right directions and Volterra's principle still remain a remarkable example of that. Another benefit is that many properties of the models can be made visible in a geometric way to scientists with little or limited background in mathematics.

Today more is known about species response to competition, nutrient limitation, predation and what environments support longer food-chains. In this paper we show how typical communities like competing species, predator-prey systems, and food-chains reply to changes in the environmental parameters.
\end{abstract}

\section{KEY WORDS:}

Deterministic modelling, intra- and interspecific competition, predatorprey model, Gause system.

AMS subject classification 92D40

\section{INTRODUCTION}

Typical modelling problems fall usually into two categories: Direct problems and inverse problems. In the first case we usually start by assuming some mechanisms that governs the observed dynamics. We then tend to formulate a model and predict dynamical scenarios. These dynamical scenarios provide usually predictions for the long-term behavior of the system under consideration under different environmental conditions, including environmental conditions not tested so far. The validity of the model and whether the right governing processes were chosen are then validated according to the model's ability to predict approximate scenarios right following, for instance, 
geographical gradients (cf. Hanski, Hansson, Henttonen (1991), Oksanen, Fretwell, Arruda, and Niemelä (1981). Of course, consistent predictions are not sufficient for validating the model and several mechanisms can yield basically the same answers.

In the second case, we tend to start from the observations and typically fit a model using "leave-one-out cross-validation" or some other process that mimics the process of learning or training the model to predict, on a short time-scale, what is going to happen. The model can be a regression spline, a neural net or some other statistically fitted model (cf. Green and Silverman (1994)). Since these models are optimized in order to yield the best possible short-term prediction, these models are superior to the former ones in this respect. Of course, something is lost and we have to be aware of that these models can predict what they are trained for only. Consequently, our possibilities to predict the response of an unknown system to a changed environmental situation is limited.

Two key questions arise in this context. The former models predicted long-term behavior from assumed mechanisms and we can ask whether we may predict mechanisms or longterm behavior from data using models optimized for short-term prediction. Predicting the governing mechanisms directly from data is the inverse problem referred to above. It turns out to be considerably more difficult than the direct problem and the best results are available in scientific areas were experiments can be repeated time after time under the same conditions, for instance physics. In ecology, already Darwinian evolution excludes the possibility for repeating an experiment under same conditions and in economy publication of new results itself will change the environmental setting and the behavior of the actors. These difficulties have not prevented scientists from making statements regarding levels of density dependence and other mechanisms goveming ecological behavior, see e.g. Saitoh, Stenseth, and Bjørnstad (1997) and Stenseth, Falck, Bjørnstad, and Krebs (1997).

Sugihara and May (1990) and Takens (1991) pointed out that there may be possibilities to make some statements regarding the long-term behavior of a given time series and we point out that this problem has turned out to be more difficult than first believed, see Morris (1990) and Nayfeh and Balachandran (1995). Even in the case of purely deterministic time series this problem may contain large difficulties because of the results by Newhouse (1979) that show that long periodic orbits with potentially small domains of attraction are abundant and common. Hence, an observed trajectory that looks chaotic may be a combination of several attracting periodic orbits and jumps between the different periodic orbits. Another difficulty is that typical motion in food-chains displays a mixture between low- and high frequency behavior and consequently very long observations are required in order to get appropriate information concerning the longterm behavior from time-series data, cf Lindström (2002) and Kuznetsov, DeFeo, and Rinaldi (2001). Also here, several attempts to make statements regarding the long-term behavior exists anyway, see Ellner and Turchin (1995) and references therein.

\section{STOCHASTIC VERSUS DETERMINISTIC}

This paper is concemed mainly with deterministic models and we think it is essential to know something about the relation between stochastic and deterministic models before 
KALMAR ECO-TECH'03

Bioremediation and Leachate Treatment

KALMAR, SWEDEN, November 25-27, 2003

proceeding with the deterministic case. First, there are many ways to include stochasticity in models. A first suggestion may be to include stochastic processes as mechanisms precisely as other mechanisms can be incorporated in the deterministic case. In this case we usually end up with models explaining when the deterministic approximation is valid (e.g. for large populations) and shows how deterministic results should be interpreted in a stochastic world. The stochastic case is mathematically considerably more difficult than the deterministic case and precise results are available for very simple models only, like the logistic growth equation, see Nåsell (2001). Yet, such approaches yield valuable information of how persistence results could be interpreted appropriately: Large populations in the deterministic persistence regime persist for finite long times (like length of universe) whereas large populations in the deterministic extinction regime persist on a short time scale only. Of course, there are not yet precise results available justifying analogous results for more complicated models than the logistic growth model and persistence questions for more complicated models yield difficult mathematical problems already in the deterministic case, see Waltman (1992).

The second way to include stochasticity is to let some parameters act as stochastic variables and this way of including stochasticity provides a platform for Monte Carlo simulations and operations research. The difficulties that will emerge can then vary depending on the chosen set of parameters. For instance, it is easier to analyze a logistic growth model with a stochastic growth rate parameter than one with stochastic carrying capacity parameter, see e. g. Lungu and Øksendal (1997). One has to include the stochasticity in a way that does not allow positive biological parameters to get negative realizations, cf Vellekoop and Högnäs (1997) and Lindström (1999), otherwise the results can yield surprises. One of the most famous current descriptions of experimental populations with simple deterministic processes falls into this cathegory, see Cushing, Dennis, Desharnais, and Costantino (1996).

A third way to include stochasticity is to let it describe the deviation between the model and the data or the part of the process that we failed to describe with a deterministic model. An attempt in this direction is given in Stenseth, Falck, Bjørnstad, and Krebs (1997).

\section{VOLTERRA'S PRINCIPLE}

A striking demonstration of the power of simple deterministic models to show directions is Volterra's principle and we shall explain this remarkable result below. To derive the result we start from the Lotka (1925) and Volterra (1926) model. Let $x$ be the density of a prey and $y$ be the density of its predator. The Lotka-Volterra model is given by the equations

$$
\begin{aligned}
& \dot{x}=r x-a x y, \\
& \dot{y}=\max y-\delta y .
\end{aligned}
$$

Its parameters are as follows: $r$ is the growth rate of the prey, $a$ is the search rate of the predator, $m$ is the conversion factor between consumed prey biomass and new predator 
biomass. Finally, $\delta$ is the death rate of the predator. Four processes have been assumed in the model and they are

(i) The prey grows exponentially in the absence of predators,

(ii) The predator is unsaturated and does not need time for digestion as it encounters one prey,

(iii) A proportion of the consumed biomass is transformed into predator biomass,

(iv) The predator decays exponentially in absence of prey (natural death).

The system has many well-known dynamical properties and we restrict our attention to those present in the positive quadrant. The terms used below are natural and specified in, for instance, in Guckenheimer and Holmes (1983). It has two fixed points (equilibrium solutions), one at the origin and and a second one at $\left(\frac{\delta}{m a}, \frac{r}{a}\right)$. The origin is a hyperbolic saddle and its stable manifold is located at the positive $y$-axis. The second fixed point is a center. Its unstable manifold is located at the positive $x$-axis. The rest of the solutions of the Lotka-Volterra system displays cyclic behavior. The system has the first integral

$$
V(x, y)=m a \int_{\frac{\delta}{m a}}^{x} \frac{x^{\prime}-\frac{\delta}{m a}}{x^{\prime}} d x^{\prime}+a \int_{\frac{r}{a}}^{y} \frac{y^{\prime}-\frac{r}{a}}{y^{\prime}} d y^{\prime}
$$

which remain invariant along all the cyclic solutions. Volterra's principle asserts that the time averages of the densities remain constant along all cyclic solutions curves and this constant equals the equilibrium values (cf. Hofbauer and Sigmund (1988)). The conclusion of this result is striking: it means that reducing the growth rate of the prey (for instance with a pesticide) will reduce the average number of the predators, not the the average number of prey! Of course, the right mechanisms are not fully included or exactly interpreted in this model but the results still show how important the analysis of nonlinear models is in showing right directions. The Lotka-Volterra model and its first integral (1) describing its cyclic solutions are also of vital importance for the analysis of more realistic models. Many results have been obtained more or less by comparing the solutions of a more realistic system to the cyclic solutions of the Lotka-Volterra system.

\section{THE GAUSE (1934) SYSTEM}

Gause (1934) made a quite useful modification of the Lotka-Volterra system. He assumed a saturated predator and competition among prey (logistic growth). In the differential equation case more processes are easy to add to the model since different processes operate independently on an infinitedecimal time-scale, see Metz and Diekmann (1986). The Holling (1959) description of saturation effects is probably still one of the best ones and the results is 
KALMAR ECO-TECH'03

Bioremediation and Leachate Treatment

KALMAR, SWEDEN, November 25-27, 2003

$$
\begin{aligned}
& \dot{x}=r x\left(1-\frac{x}{K}\right)-y \frac{a x}{1+a b x}, \\
& \dot{y}=m y \frac{a x}{1+a b x}-\delta y .
\end{aligned}
$$

The variables, $x$ and $y$, are still the prey and predator densities, respectively, and the parameters $r, a, m$, and $\delta$ are as above. The two newly introduced parameters are the environmental carrying capacity $K$ that corresponds to the enrichment of the system and the predator's handling time $b$. The handling time is the average time the predator stops searching for new prey as it encounters one. The function

$$
f(x)=\frac{a x}{1+a b x}
$$

above is called the functional response of the predator. It measures the number of prey caught per predator per unit of time as a function of prey-density. Gause asserted that every saturated predator should have a bounded functional response. The particular case (2) above is called the Holling II functional response among ecologists and the MichelisMenten law among scientists with background in chemistry. The logistic growth equation with its quadratic term $-x^{2} / K$ is considerably more controversial than the other terms in the above model (see e. g. Kooi, Boer, and Kooijman (1998) and Domingo, Biebricher, Eigen, Holland (2001)). In the case above we can interpret the prey growth as something proportional to both prey density and space left

The long-term dynamics of the Gause model is quite different from the corresponding dynamics of the Lotka-Volterra model that essentially displayed the same dynamics for all parameter values. For low enrichment levels (low $K$ ), the predator goes extinct and the prey density tends to the environmental carrying capacity (logistic growth). For intermediate enrichment levels both species coexist in equilibrium. Limit cycles can be excluded by several methods and one of them is comparing the solutions to the closed orbits of the Lotka-Volterra system (1), see Lindström (1989), and another one is Dulac's criterion (Hsu, Hubbell, and Waltman (1978)). Both species are persistent. For higher enrichment levels the coexistence is oscillatory and both the period and the amplitude of the cycles increase with enrichment. The system becomes less persistent and more sensitive to perturbations. It is interesting that it is possible to prove the uniqueness of limit cycles for the Gause-system above. There are many proofs of this assertion available, but the best approach in this direction is still that of Kuang and Freedman (1988). The problem of determining upper bounds for the number of limit cycles for differential equations in the real plane is related to the famous Hilbert's 16th problem (cf. Smale (1998)).

\section{EXAMPLES OF DIFFICULT AND IMPORTANT COMPETITION PROBLEMS IN THE APPLIED SCIENCES}

Competition is a key ingredient in population dynamics. There is a potential to handle many real world problems successfully by an adequate understanding of the competition 
KALMAR ECO-TECH'03

Bioremediation and Leachate Treatment

KALMAR, SWEDEN, November 25-27, 2003

processes that takes place. We commence by taking three examples ranging from composting and toxic algae blooms to nanoevolution of viruses here.

Competition for nutrients has since long been the explanation for the selection and succession of phytoplankton species. Traditional competition theory predicts that the number of existing phytoplankton species cannot exceed the number of limiting nutrients under steady-state conditions (Tilman (1982)). Considering the relative homogenous environment of plankton communities, and the few possible limiting nutrients, the enormous diversity of phytoplankton in natural waters has been regarded as the "plankton paradox" (Hutchinson (1961)). Several solutions to this paradox have been proposed, many of them based on modelling of intraspecies competition. Deterministic modelling have been used to show the coexistence of a larger number of species through internal oscillations, as one of the solutions of the plankton paradox (Huisman and Weissing (1999)). Non-steady state conditions have also been used to explain the coexistence of a larger number of species than the number of limiting resources (e.g. Scheffer et al. (2003)). Deterministic models have further been used to explain the dominance of larger diatoms under fluctuating nutrient conditions when nitrate is the prevailing nitrogen source, in agreement with observations in marine coastal waters (Stolte and Riegman (1996)). Concerning the environmental problem of harmful algal blooms, plankton models combining herbivory and competition have been used to explain the role of eutrophication in the development of poorly edible, harmful or even toxic phytoplankton species (Riegman and Kuipers (1994), and Grover (1995)).

Clonal expansion of competing variants has been described for several viruses including the human immunodeficiency virus (HIV), foot-and-mouth disease virus and vesicular stomatitis virus (see e. g. Domingo, Baranowski, Escarmís, and Sobrino (2002), Domingo, Escarmis, Baranowski, Ruiz-Jarabo, Carillo, Nunez, and Sobrino (2003), Moya, Elena, Bracho, Miralles, and Barrio (2000), Rambaut, Posala, Crandall, and Holmes (2004), Rouzine and Coffin (1999), and Rouzine, Rodrigo, and Coffin (2001)). It has been shown that due to the high mutation and recombination rate, RNA viruses exist and easily do not become extinct during competition in a multicellular organism. There is however no preservation or stasis in their status. Instead, RNA viruses are constantly changing and the dynamic behavior is not only observed in a complex environment where pressure of the immune system and need to adapt to various cell types force the viral population to exert optimal changing, but also in a simple environment such as a homogenous culture of in vitro cultivated mammal cells. In finding successful strategies for treatment like highly active antiretroviral therapy (HAART), one have to get an acceptable understanding of the governing mechanisms for competition for each viral agent (see e. g. Rambaut et.al. (2004)). Today, it is also known that evolutionary suicide can be the outcome under certain conditions, see Gyllenberg, Parvinen, and Dieckmann (2002).

Composting is one of the most complex natural biotechnologies. It has been applied to various applications successfully ranging from waste reduction to food production. This method also causes a myriad of physical and biological states that are unexpected and unwanted, odor formation, incomplete stabilization, disruption in the degradation processes, and poor experimental reproducibility are the manifestations of these 
KALMAR ECO-TECH'03

Bioremediation and Leachate Treatment

KALMAR, SWEDEN, November 25-27, 2003

unexpected states (Schloss, Hay, Wilson, and Walker (2003)). Composting is a process driven by microbial community succession, but there is only a limited amount of qualitative data in this regard. It is important to mention that a large variety of mesophilic thermotolerant and thermophilic aerobic microorganisms including bactia actinomycetes, yeast and various other fungi have been found in compost and other self heating organic materials. There are many factors that determine the microbial conditions. Temperature is the major factor that determines the types of microorganisms, species diversity, and the rate of metabolic activities (Hassen, Belguith, Jedidi, Cherif, Cherif, and Boudabous (2001)).

Aerobic organisms thrive at oxygen levels greater than 5 percent (air is about 21 percent oxygen). They are the preferred microorganisms since they provide the most rapid and effective composting. Anaerobic microorganisms thrive when the compost pile is oxygen deficient. Anaerobic conditions are undesirable. The products of anaerobic decomposition cause compost piles to smell badly. Aerobic bacteria are the most important initiators of decomposition and temperature increase within the compost pile. Several types of bacteria thrive between the temperatures of 15-70. The initial temperature of the compost pile is usually related to air temperature. At temperatures below 21, helpful bacteria do not thrive.

While high temperatures 60 kill most pathogenic organisms and weed seeds, the most effective decomposing bacteria are those that grow at moderate temperatures 21-38. Temperature changes during the process depend on materials being composted, compost method and the water available. Pile temperatures between 32-70 indicate rapid decomposition. The management of the compost process is the determining factor in the destruction of weed seeds, disease organisms and other pathogens. Well-managed systems result in excellent control. Ill-managed systems result in an inconsistent product. (Romantschuk, Sarand, Petänen, Peltola, Jonsson-Vihanne, Koivula, Yrjälä, and Haahtela (2000), Miller and Clesceri (2003)).

Thus to achieve a well managed system, it is imperative that knowledge and understanding of the system is obtained, this knowledge can be obtained by finding the adequate competition model. In order to be adequate, a potential model must include major classes of bacteria and must be able to predict dominant classes physical and biological final states.

\section{COMPETITION MODELS IN CONTINUOUS AND DISCRETE TIME}

The logistic equation

$$
\dot{x}=r x\left(1-x / K^{\prime}\right)
$$

assumes that the growth rate of a given species is proportional to the space left and the population density. The species is basically limited by some stable resources like territories (for reproduction) or refuges (for survival), cf Hassell (1976). We have assumed overlapping generations and want to find a model that models the same processes for non-overlapping generations. Typical species possessing non-overlapping 
KALMAR ECO-TECH'03

Bioremediation and Leachate Treatment

KALMAR, SWEDEN, November 25-27, 2003

generations are terrestrial arthropods in boreal environments, see Borror, DeLong, and C. A. Triplehorn (1976). We then assume that the death processes remain continuous whereas the birth processes are discrete, see e. g. Brauer and Castillo-Chávez (2001) Gyllenberg, Hanski, and Lindström (1996,1997), Lindström (1999,2002,2003). The death processes consist of natural death and the logistic competition term:

$$
\dot{x}=\underbrace{-\mu_{1} x}_{\text {natural death }} \overbrace{-\mu_{2} x^{2}}^{\text {logisticcompetition term }} .
$$

The nonlinear (Bemoulli) equation above can be solved through separation of variables and we get

$$
x(t)=\frac{x_{0} \Subset x p\left(-\mu_{1} t\right)}{1+\frac{\mu_{2}}{\mu_{1}} x_{0}\left(1-\exp \left(-\mu_{1} t\right)\right)}
$$

where $x_{0}$ is a given initial population density and $x(t)$ are the remaining reproductive individuals density. We let these individuals multiply with the factor $\beta_{0}$ at time $t=T$ and get a density of $\beta_{0 x}(T)$ in the beginning of the next season. That is,

$$
x(T)=\frac{\beta_{0} x_{0} \exp \left(-\mu_{1} T\right)}{1+\frac{\mu_{2}}{\mu_{1}} x_{0}\left(1-\exp \left(-\mu_{1} T\right)\right)}
$$

We put $\beta=\beta_{0} \exp \left(-\mu_{I} T\right)$ and $X_{\tau}=\frac{\mu_{2}}{\mu_{1}} x_{0}\left(1-\exp \left(-\mu_{1} T\right)\right)$. We get the discrete model

$$
X_{\tau+T}=\frac{\beta X_{\tau}}{1+X_{\tau}}
$$

and we can assume $T=1$ without loss of generality so that

$$
X_{\tau+1}=\frac{\beta X_{\tau}}{1+X_{\tau}} .
$$

The model (4) is the Beverton-Holt (1957) model, a discrete correspondence to the logistic growth equation (3). It is remarkable that these two models have similar dynamics, for parameters describing a persistent organism, both models have two fixed points, the origin is unstable, and the equilibrium corresponding to the carrying capacity is globally stable for all positive initial population densities. It is even more remarkable that there are possibilities for detecting corresponding dynamical similarities in longer food chains, see Lindström (2002).

\section{COMPETITION TYPES}

Discrete single species models can describe competition types that cannot be described by continuous single species models. The function describing the right hand-side of (4) 
was strictly increasing, but we could in principle allow for right-hand sides that both increases and decreases. Nicholson (1954) classified intraspecific competition into two basic types: contest and scramble. In their extreme form these two types are modelled by

$$
X_{\tau+1}=\left\{\begin{array}{cc}
\beta X, & \beta X<K \\
K, & \beta X>K
\end{array}\right.
$$

and

$$
X_{\tau+1}=\left\{\begin{array}{cc}
\beta X, & \beta X<K \\
0, & \beta X>K
\end{array},\right.
$$

respectively. In the first case the resources are extremely unequally shared. A portion of individuals get all they require for reproduction, whereas the rest get insufficient for survival and reproduction. This type of competition leads to stable dynamics, and we already noted that the equilibrium position at the carrying capacity was globally asymptotically stable in the logistic case.

In the other type (scramble), resources are equally shared and fast local growth followed by local crashes are predicted. This type of competition is typical when rather transient resources are competed for. We can, for instance, regard viruses as predators on their target cells. In simple systems such as a bottle of cultured cells, the virus uses the cell metabolism (feeds on the cells) to synthesize new virus particles until all cells are destroyed. This process is comparable to a viral infection where the infected organism dies. Another event may be that the virus may not be able to infect and destroy all cells and therefore resistant cells will be selected and divide and replace the susceptible cells. The process may be compared to the immune response that clears the virus from the organism. In both cases it leads to a local catastrophe for the virus in question. RNA virus replicating in multicellular hosts need however to invade and successfully replicate in tissues that comprise a variety of cell types. The highly variable mutant cloud of viruses will facilitate replication and spread in this heterogeneous environment and the dynamics of these complex interactions may be modelled (Cuevas, Moya, and Elena (2003)). A virus must develop or evolve before the the immune system replies globally, and the transience and heterogeneity of the resources are generally the factors that drive development further.

The first type of competition promotes defence whereas the second promotes development. In most cases competition is a mixture of these two types of competition, and the logistic competition (Beverton-Holt) is a competition type where contest type factors dominate. It is interesting to note that contest type discrete models with strictly increasing right-hand sides can be derived from single species non-structured continuous models, but scramble type discrete cannot. The reason is the uniqueness of solutions theorem for ordinary differential equations in the fixed reproductive strategy case (cf. Clark (1990)). This result can be extended to to the adjustable reproductive strategy case, see Gyllenberg et. al (1997). 


\section{COMPETITION OUTCOME: EXAMPLE 1}

Consider two types of the same organism, both have different birth factors, clonal reproduction, and affect the environment in the same way. However, they experience the environment and crowding effects differently. They compete in a scramble type way described by the Ricker (1954) equation

$$
\begin{aligned}
& x_{t+1}=\beta_{1} x_{t} \exp \left(-k_{1}\left(x_{t} \mathrm{e}+\mathrm{e} y_{t}\right)\right) \\
& y_{t+1}=\beta_{2} y_{t} \exp \left(-k_{2}\left(x_{t}+y_{t}\right)\right)
\end{aligned}
$$

Here, $\beta_{1}$ and $\beta_{2}$ are the birth factors (clutch sizes) of the two types $x_{t}$, and $y_{t}$ respectively. The constants $k_{l}$ and $k_{2}$ measures how the two types experience crowding, respectively. We could expect a trade-off between the reproductive effort and the competitive ability, but we do not need to assume that in order to obtain complete results for the model (7). In fact, the species possessing the highest value of $\log \beta_{i} / k_{i}, i=1,2$ will outcompete the other species (see Gyllenberg et. al. (1996)). The parameter value $\log \beta_{i} / k_{i}$ corresponds exactly to the mean population density of the type in the absence of the other. Hence. the species possessing the highest mean density will outcompete the other regardless of whether the predicted dynamics is stable or not. This is called Fisher's (1930) maximum principle. Since one of the species generically outcompetes the other one, we also say that the principle of competitive exclusion (Hardin (1960)) holds for this model. In the experimental literature this principle is called Gause's principle after that Gause (1934) took the results by Volterra (1926) and Lotka (1932) as a basis for his testing of the principle. There are a rich variety of experimental systems obeying this principle, for instance, when competing viruses of the same or related kind co-exist there will be competition that will finally result in the victory of one type (Moya et. al. (2000)).

\section{COMPETITION OUTCOME: EXAMPLE 2}

As a last example we take the following model describing two predators competing for the same prey (Hsu et. al. (1978)):

$$
\begin{gathered}
\dot{s}=r s(1-s / K)-\frac{a_{1} x_{1} s}{1+a_{1} b_{1} s}-\frac{a_{2} x_{2} s}{1+a_{2} b_{2} s} \\
\dot{x_{1}}=\frac{m_{1} a_{1} x_{1} s}{1+a_{1} b_{1}}-\delta_{1} x_{1} \\
\dot{x_{2}}=\frac{m_{2} a_{2} x_{2} s}{1+a_{2} b_{2} s}-\delta_{2} x_{2}
\end{gathered}
$$

Here, the prey density is given byes and the density of the two predators is given by $x_{l}$ and $x_{2}$, respectively. The prey grows according to the logistic law, and both predators harvest the prey according to the Holling II functional response. Consider now the two systems 


$$
\begin{aligned}
\dot{s} & =r s(1-s / K)-\frac{a_{1} x_{1} s}{1+a_{1} b_{1} s} \\
\dot{x_{1}} & =\frac{m_{1} a_{1} x_{1} s}{1+a_{1} b_{1} s}-\delta_{1} x_{1},
\end{aligned}
$$

and

$$
\begin{aligned}
\dot{s} & =r s(1-s / K)-\frac{a_{2} x_{2} s}{1+a_{2} b_{2} s} \\
\dot{x_{2}} & =\frac{m_{2} a_{2} x_{2} s}{1+a_{2} b_{2} s}-\delta_{2} x_{2},
\end{aligned}
$$

separately. Each of these systems can be dynamically classified to either, predator extinction, stable coexistence, or oscillatory coexistence. Suppose now that both (9) and (10) possess stable coexistence, the predator extinction case does not make sense here. In that case the principle of competitive exclusion remains valid and the predator species with lower value of the parameter

$$
\lambda_{i}=\frac{\delta_{i}}{a_{i}\left(m_{i}-b_{i} \delta_{i}\right) \mathrm{t}}, i=1,2
$$

outcompetes the other species, see Chiu (1999) and Lindström (2000). Competitive exclusion holds in many other cases, too, but system (8) possesses known violations of this principle (cf. Muratori and Rinaldi (1989), McGehee and Amstrong (1977), and Keener (1983), Osipov, Söderbacka, and Eirola (1996)). The first one to construct an experimental system violating the principle of competitive exclusion was Utida (1957).

Even though Fisher's maximum principle holds in some cases for the system (8), there are cases even in the situation where both systems (9) and (10) possess equilibrium coexistence violating this principle. More precisely, this can be seen as follows: The parabolas

$$
x_{i}(s)=\frac{r}{a_{i}}\left(1-\frac{s}{K}\right)\left(1+a_{i} b_{i} s\right), i=1,2
$$

can be sketched in the same coordinate system and if they do not coincide, they can intersect each other in at most two points. If we put $a=a_{1}=a_{2}$, then the intersection points are $(K, 0)$ and $(0, r / a)$. We may now choose $b_{I}<b_{2}$ and this ensures that $x_{I}(s)<$ $x_{2}(s)$ for $0<s<K$. We may now choose $\delta_{i}=1 / b_{i}, i=1,2$. We can still solve for all positive values of $\lambda_{i}, i=1,2$ by choosing $m_{i}, i=1,2$. Since the parameter $\lambda_{i}$ determined the winner we can choose both winners that support the principle and winners that violate it. In the oscillatory case with valid competitive exclusion, the situation is even worse. Since a typical winner has a low value of $\lambda_{i}$ and saddle points exist at the origin and at $(K$, $0,0)$, the strongly oscillatory trajectories will spend a lot of time close to these points, forcing the mean abundance of the winning type close to zero. Hence, no concise answer about what traits are enhanced by evolution exists today. 


\section{SUMMARY}

I this paper we have discussed different modelling strategies and possible problems that may arise. The scope has been to point out what problems may be relevant in the interface between mathematics and biological sciences. We have given examples of two quite well-known artificial competition systems that give very diverging predictions for evolving systems. These systems show that it is not well-known today what patterns and traits generally are promoted by evolution. We have linked the mathematical presentation to several important real-world systems ranging from toxic algae blooms and RNA virus evolution to compost control systems and have highlighted the importance of a continued open search for common patterns in such systems.

\section{REFERENCES}

[1] R. J. H. Beverton and S. J. Holt. On the Dynamics of Exploited Fish Populations, volume 19 of Fisheries Investigation Series 2. Ministry of Agriculture, Fisheries and Food,eLondon, 1957.

[2] D. J. Borror, D. M. DeLong, and C. A. Triplehorn. An Introduction to the Study of Insects. Holt, Rinehart, and Winston, fourth edition, 1976.

[3] F. Brauer and C. Castillo-Chávez. Mathematical Models in Population Biology and Epidemiology, volume 40 of Texts in applied mathematics. Springer, 2001.

[4] C.-H. Chiu. Lyapunov functions for the global stability of competing predators. Journal of Mathematical Analysis and Applications, 230:232-241, 1999.

[5] C. W. Clark. Mathematical Bioeconomics. John Wiley \& Sons, second edition, 1990.

[6] J. M. Cuevas, A. Moya, and S. F. Elena. Evolution of ma virus in spatially heterogenous environments. J. Evol. Biol, 16:456-466, 2003.

[7] J. M. Cushing, B. Dennis, R. A. Deshamais, and R. F. Costantino. An interdisciplinary approach to understanding nonlinear ecological dynamics. Ecological Modelling, 92:111-119, 1996.

[8] C. Darwin. On the origin of species by means of natural selection. John Murray, London, sixth edition, 1878. Cited from 1917 printing.

[9] E. Domingo, E. Baranowski, C. Escarmís, and F. Sobrino. Foot-and-mouth disease virus. Comparative Immunology, Microbiology \& Infectious Diseases, 25:297-308, 2002.

[10] E. Domingo, C. K. Biebricher, M. Eigen, and J. J. Holland. Quasispecies and RNA Virus Evolution: Principles and Consequences, volume 14 of Molecular biology intelligence unit. Landes Bioscience, 2001.

[11] E. Domingo, C. Escarmis, E. Baranowski, C. M. Ruiz-Jarabo, E. Carillo, J. I. Nunez, and F. Sobrino. Evolution of the foot-and-mouth disease virus. Virus Res, 91:47-63, 2003.

[12] S. Ellner and P. Turchin. Chaos in a noisy world: New methods and evidence from time-series analysis. The American Naturalist, 145(3):343-375, 1995. 
KALMAR ECO-TECH'03

Bioremediation and Leachate Treatment

KALMAR, SWEDEN, November 25-27, 2003

[13] R. A. Fisher. The Genetical Theory of Natural Selection. Clarendon Press, Oxford, 1930.

[14] G. F. Gause. The Struggle for Existence. The Williams \& Wilkins Company, Baltimore, 1934.

[15] P. J. Green and B. W. Silverman. Nonparametric regression and Generalized Linear Models, volume 58 of Monographs on Statistics and Applied Probability. Chapman \& Hall, 1994.

[16] J. P. Grover. Competition, herbivory, and enrichment: nutrient based models for edible and inedible plants. The American Naturalist, 145:746-774, 1995.

[17] J. Guckenheimer and P. Holmes. Nonlinear Oscillations, Dynamical Systems, and Bifurcations of Vector Fields. Springer-Verlag, 1983.

[18] M. Gyllenberg, I. Hanski, and T. Lindström. A predator-prey model with optimal suppression of reproduction in the prey. Mathematical Biosciences, 134:119-152, 1996.

[19] M. Gyllenberg, I. Hanski, and T. Lindström. Continuous versus discrete single species population models with adjustable reproductive strategies. Bulletin of Mathematical Biology, 59(4):679-705, 1997.

[20] M. Gyllenberg, K. Parvinen, and U. Dieckmann. Evolutionary suicide and evolution of dispersal in structured metapopulations. Journal of Mathematical Biology, 45:79- 105, 2002

[21] I. Hanski, L. Hansson, and H. Henttonen. Specialist predators, generalist predators, and the microtine rodent cycle. Journal of Animal Ecology, 60:353-367, 1991.

[22] G. Hardin. The competitive exclusion principle. Science, 131:1292-1297, 1960.

[23] M. P. Hassell. The Dynamics of Competition and Predation. The Camelot Press Ltd, Southampton, 1976.

[24] A. Hassen, K. Belguith, N. Jedidi, A. Cherif, M. Cherif, and A. Boudabous. Microbial characterization during composting of municipal solid waste. Bioresource technology, 80:217-225, 2001.

[25] J. Hofbauer, K. Sigmund. The theory of evolution and dynamical systems. Cambridge University Press, Cambridge, 1988.

[26] C. S. Holling. Some characteristics of simple types of predation and parasitism. The Canadian Entomologist, 91(7):385-398, 1959.

[27] S. B. Hsu, S. P. Hubbell, and P. Waltman. Competing predators. SIAM Joumal of Applied Mathematics, 35(4):617-625, 1978.

[28] J. Huisman and F. J. Weissing. Biodiversity of plankton by species oscillations and chaos. Nature, 402:407-410, 1999.

[29] G. E. Hutchinson. The paradox of plankton. The American Naturalist, 95:137-145, 1961.

[30] J. P. Keener. Oscillatory coexistence in the chemostat. SIAM Journal of Applied Mathematics, 43(5):1005-1018, 1983. 
[31] B. W. Kooi, M. P. Boer, and S. A. L. M. Kooijman. On the use of the logistic equation in models of food chain. Bulletin of Mathematical Biology, 60:231-246, 1998.

[32] Y. Kuang and H. I. Freedman. Uniqueness of limit cycles in Gause-type models of predator-prey systems. Mathematical Biosciences, 88:67-84, 1988.

[33] Y. A. Kuznetsov, O. De Feo, and S. Rinaldi. Belyakov homoclinic bifurcations in a tritrophic food chain model. SIAM Journal of Applied Mathematics, 62(2):462-487, 2001.

[34] T. Lindström. A generalized uniqueness theorem for limit cycles in a predator-prey system. Acta Academiae Aboensis, Ser B, 49(2):1-9, 1989.

[35] T. Lindström. Dependencies between competition and predation - and their consequences for initial value sensitivity. SIAM Journal of Applied Mathematics. 59(4):1468-1486, 1999.

[36] T. Lindström. Global stability of a model for competing predators: the Ardito \& Ricciardi Lyapunov functional. Nonlinear Analysis, 39:793-805, 2000.

[37] T. Lindström. On the dynamics of discrete food-chains: Low- and high-frequency behavior and chaos. Journal of Mathematical Biology, 45:396-41 8, 2002.

[38] T. Lindström. Discrete models and fisher's maximum principle in ecology. In Discrete and Continuous Dynamical Systems, Expanded volume, Proceedings of the Fourth International Conference on Dynamical Systems and Differential Equations, May 24-27, 2002, Wilmington, NC, USA, to appear 2003.

[39] A. J. Lotka. Elements of Physical Biology. Williams and Wilkins, Baltimore, 1925.

[40] A. J. Lotka. The growth of mixed populations: Two species competing for a common food supply. Journal of the Washington Academy of Sciences, 22:461-469, 1932.

[41] E. M. Lungu and B. Øksendal. Optimal harvesting from a population in a stochastic crowded environment. Mathematical Biosciences, 145:47-75, 1997.

[42] T. R. Malthus. An essay on the principle of population (and: A summary view of the principle of population. Penguin, Harmondsworth, Middlesex, 1798, 1970.

[43] R. McGehee and R. A. Armstrong. Some mathematical problems conceming the ecological principle of competitive exclusion. Journal of Differential Equations, 23:3052, 1977.

[44] J. A. J. Metz and O. Diekmann. The Dynamics of Physiologically Structured Populations. Springer-Verlag, 1986

[45] A. P. Miller and N. L. Clesceri. Waste sites as biological reactors: Characterization and modelling. Kinetics of Decomposition of wastes, pages 229-290. Lewis Publishers, CRC Press LLC, 2003.

[46] W. F. Morris. Problems in detecting chaotic behavior in natural populations by fitting simple discrete models. Ecology, 71(5):1849-1862, 1990. 
KALMAR ECO-TECH'03

Bioremediation and Leachate Treatment

KALMAR, SWEDEN, November 25-27, 2003

[47] A. Moya, S. F. Elena, A. Bracho, R. Miralles, and E. Barrio. The evolution of ma viruses: A population genetics view. Proceedings of the National Academy of Sciences of the United States of America, 96:6967-6973, 2000.

[48] S. Muratori and S. Rinaldi. Remarks on competitive coexistence. SIAM Journal of Applied Mathematics, 49(5):1462-1472, 1989.

[49] I. Nåsell. Extinction and quasi-statinarity in the Verhulst logistic model. Journal of Theoretical Biology, 211(1):11-27, 2001.

[50] A. H. Nayfieh and B. Balachandran. Applied Nonlinear Dynamics. John Wiley \& Sons, 1995.

[51] S. N. Newhouse. The abundance of wild hyperbolic sets and non-smooth stable sets for diffeomorphisms. Publ. Math. IHES, 50:349-399, 1979.

[52] A. J. Nicholson. An outline of the dynamics of animal populations. Australian Journal of Zoology, 2:9-65, 1954.

[53] L. Oksanen, S. D. Fretwell, J. Arruda, and P. Niemelä. Exploitation ecosystems in gradients of primary productivity. The American Naturalist, 118(2):240-261, 1981.

[54] A. V. Osipov, G. Söderbacka, and T. Eirola. On the appearance of chaotic solutions in a dynamical system of two predators - one prey type. Actual Problems in Contemporary Mathematics, 1:39-70, 1996. ISBN 5-7320-0421-1, in Russian, English abstract.

[55] A. Rambaut, D. Posala, K. A. Crandall, and E. C. Holmes. The causes and consequences of hiv evolution. Nature Reviews Genetics, 5:52-61, 2004.

[56] W. E. Ricker. Stock and recruitment. Journal of the Fisheries Research Board of Canada, 11(5):559-623, 1954.

[57] R. Riegman and B. R. Kuipers. Resource competition and selective grazing of plankton in a multispecies pelagic food web model. Mar. Ecol., 15:153-164, 1994.

[58] M. Romantschuk, I. Sarand, , T. Petänen, R. Peltola, M. Jonsson-Vihanne, T. Kuivula, K. Yrjälä, and K. Haahtela. Means to improve the effect of insitu bioremediation of contaminated soil: an overview of novel approaches. Environmental Pollution, 107:179-185, 2000.

[59] I. M. Rouzine and J. M. Coffin. Interplay between experiment and theory in devepoment of a working model for hiv population dynamics. In E. Domingo, R. Webster, and J. Holland, editors, Origin and Evolution af viruses, pages 225-285. Academic Press, San Diego, 1999.

[60] I. M. Rouzine, A. G. Rodrigo, and J. M. Coffin. Transition between stochastic evolution and deterministic evolution in the presence of selection. Microbiol. Mol. Biol. Rev., 65:151-185, 2001

[61] T. Saitoh, N. C. Stenseth, and O. N. Bjørnstad. Density dependence in fluctuating grey-sided vole populations. Journal of Animal Ecology, 66:14-24, 1997.

[62] M. Scheffer, S. Rinaldi, J. Huisman, and F. J. Wiessing. Why plankton communities have no equilibrium: solutions to the paradox. Hydrobiologia, 491:9-18, 2003. 
KALMAR ECO-TECH'03

Bioremediation and Leachate Treatment

KALMAR, SWEDEN, November 25-27, 2003

[63] P. D. Schloss, A.eG. Hay, D. B. Wilson, and L. P. Walker. Source tracking temporal changes of bacterial community fingerprints during initial states of composting. FEMS Microbiology Ecology, 46:1-9, 2003

[64] S. Smale. Mathematical problems for the next century. The Mathematical Intelligencer, 20:7-15, 1998.

[65] N. C. Stenseth, W. Falck, O. Bjørnstad, and C. J. Krebs. Population regulation in snowshoe hare and Canadian lynx: Asymmetric food web configurations between hare and lynx. Proceedings of the National Academy of Sciences of the United States of America, 94:5147-5152, 1997.

[66] W. Stolte and R. Riegman. A model approach for size-selective competition of marine phytoplankton for fluctuating nitrate and ammonium. J. Phycol., 32:732-740, 1996.

[67] G. Sugihara and R. M. May. Nonlinear forecasting as a way of distinguishing chaos from measurement error in time series. Nature, 344:734-741, 1990.

[68] F. Takens. Detecting strange attractors in turbulence. In D. A. Rand and L. S. Young, editors, Dynamical Systems and Turbulence, Warwick 1980, Lecture Notes in Mathematics 898, pages 366-381. Springer-Verlag, 1981.

[69] D. Tilman. Resourse competition and community structure, volume 17. Princeton University Press, Princeton, New Jersey, 1982.

[70] S. Utida. Population fluctuation, an experimental and theoretical approach. Cold Spring Harbor Symposia on Quantitative Biology, 22:139-151, 1957.

[71] M. H. Vellekoop and G. Högnäs. A unifying framework for chaos and stochastic stability in discrete population models. Journal of Mathematical Biology, 35:557- 588, 1997.

[72] V. Volterra. Variazioni e fluttuazioni del numero d'individui in specie animali conviventi. Memorie della R. Accademia Nationale dei Lincei 6, 2:31-113, 1926.

[73] P. Waltman. A brief survey of persistence in dynamical systems. Springer Lecture notes in Mathematics, 1475:31-40, 1992. 
KALMAR ECO-TECH'03

Bioremediation and Leachate Treatment

KALMAR, SWEDEN, November 25-27, 2003

\section{POSTER SESSION}

